\title{
Intramammary Gel Dosage Form
}

National Cancer Institute

\section{Source}

National Cancer Institute. Intramammary Gel Dosage Form. NCI Thesaurus. Code C149605.

Semi-solid preparation for veterinary use consisting of a gel containing dissolved or dispersed active substance(s) intended for intramammary use. Intramammary gels are presented in intramammary syringes for use on one occasion only to introduce the gel into a single teat canal. 